\title{
The relation between discriminability and memory for vowels, consonants, and silent-center vowels
}

\author{
AIMEE M. SURPRENANT and IAN NEATH \\ Purdue University, West Lafayette, Indiana
}

\begin{abstract}
People remember lists of vowel-contrasting syllables better than lists that vary only in stop consonant identity. Most views suggest that this difference is due to the structure of immediate memory and the greater discriminability of vowels compared with consonants. In all of these views, there is a presumed systematic relationship between discriminability and recall so that the more discriminable an item, the better that item should be recalled. The 11 experiments reported here measured the relative discriminability of and compared serial recall for (1) intact syllables that varied only in the medial vowel, (2) intact syllables that varied only in the initial consonant, and (3) syllables with the center vowel replaced by silence (so-called silent-center vowels). When item discriminability, as measured by identification, was equated for consonant-contrasting and silent-center lists, serial recall performance was also equal. However, even when the vowels were less discriminable than the consonants or silentcenter vowels, serial recall performance for the vowels was still better. These results are problematic for theories based on acoustic discriminability but can be explained parsimoniously by Nairne's (1990) feature model.
\end{abstract}

Theories detailing how people briefly retain the auditory characteristics of sound have been deeply influenced by research on memory for vowels and consonants. A number of different paradigms, including categorical perception, imagery, and serial list recall, have all demonstrated impoverished performance for stop consonants compared to that for vowels (see Surprenant, 1992, for a review). Most of the explanations of these results rely on an interaction between the structure of immediate memory and the relative discriminability of the items in the stimulus set.

Several experiments have revealed differences in memory for vowels and stop consonants with a serial list recall task. In this paradigm, subjects are asked to recall a list of items in the order in which they were presented. When auditory - or vocalized - presentation is used, people generally recall the first few items well (the primacy effect) and the last few items well (the recency effect) relative to the middle items (Crowder, 1976). When visual presentation is used, there is no recency effect (LeCompte, 1992), and this difference between auditory and visual presentation is known as the modality effect (see Penney, 1989, for a review). When the to-be-remembered items are syllables that differ only in the vowel sounds, there is a particularly large and robust recency effect compared with when the

Some of this research was supported by an NIDCD Individual National Research Service Award to A.M.S., and portions of these data were presented at the 35th Annual Meeting of the Psychonomic Society, St. Louis, November 1994. We thank J. S. Nairne for comments on an earlier version of this manuscript. Correspondence should be addressed to A. M. Surprenant, 1364 Psychological Sciences Building, Purdue University, West Lafayette, IN 47907-1364 (e-mail: aimee@psych. purdue.edu). syllables differ by only a stop consonant (Cole, 1973; see also Crowder, 1971). Cole (1973) and Drewnowski (1980) also reported an advantage for vowel lists compared with stop consonant lists at early serial positions.

The results from serial recall have most often been accounted for by invoking a sensory memory system devoted to acoustic information. The most dominant theory of such an echoic memory system was precategorical acoustic store (PAS) (Crowder \& Morton, 1969). This theory assumes that auditory to-be-remembered items are stored in a relatively uncategorized code for brief periods of time, around $2 \mathrm{sec}$ or so, in a modality-specific sensory memory system. The store is said to be "precategorical" because it contains unanalyzed information, and it is "acoustic" because it retains only acoustic information, or more accurately, a representation of the acoustic information. Newly entering items interfere with already present items, but only if the new items are acoustically similar to the previous items. PAS provides a source of supplementary information that can be used to identify or recall an auditory item.

The modality effect arises because auditory presentation of verbal material permits information about the last item or two to remain in PAS, from which it can be recalled. Because PAS holds items with more steady-state information for longer periods than it does items with more transient information (Crowder, 1973b, 1975), there will be more information concerning item identity in PAS when the stimuli are vowels than when they are consonants. Thus, according to this view, vowels should be better identified than consonants because the acoustic information describing a given vowel will last longer in PAS than will the corresponding information that describes a consonant, and vowels should be recalled more accurately for similar reasons. 
PAS thus offered a single explanation for both modality and suffix effects, and also explained the better recall of vowels compared with consonants. Despite the popularity of that view, however, many studies have since demonstrated that the effects attributed to PAS are not necessarily precategorical, such as the context-dependent suffix effect (Ayres, Jonides, Reitman, Egan, \& Howard, 1979; Neath, Surprenant, \& Crowder, 1993), and need not necessarily be acoustic, such as the modality effects observed with mouthed or lip-read stimuli (Nairne \& Crowder, 1982; Spoehr \& Corin, 1978).

Although PAS is no longer the dominant account for memory effects (Surprenant, Pitt, \& Crowder, 1993), a similar explanation has been developed to account for differences in the durability of the auditory memory code for vowels and stop consonants in the categorical perception paradigm. In this paradigm, subjects participate in two phases: an identification task and a discrimination task. ${ }^{1}$ The stimuli are typically synthetic syllables constructed by changing one parameter (such as voice onset time [VOT]) in a number of equal steps between two speech stimuli. VOT is the time interval between the first release of air in pronouncing a consonant and the beginning of voicing, and it is shorter for voiced consonants than for unvoiced consonants. In a VOT continuum, each token differs from the other tokens in the duration of the VOT, which is systematically lengthened as one moves from gift to kift, for example. This results in a continuum of sounds varying in equal steps from one syllable to another. In the identification phase, subjects are asked to classify each stimulus into one of (usually) two categories; in the example above, the subjects indicate whether they heard a $/ \mathrm{k} /$ or a $/ \mathrm{g} /$. Then, subjects are given a discrimination task, typically an $A B X$ task, where the subject hears two different tokens, A and $\mathrm{B}$, and then decides if a third token, $\mathrm{X}$, is the same as $\mathrm{A}$ or the same as B.

A continuum is said to exhibit categorical perception if the stimuli within a category (as determined by the identification task) are discriminated at chance levels whereas those stimuli classified into different categories are discriminated almost perfectly, even though the physical differences between the stimuli (within and between categories) are the same. With this task, it is generally found that continua of stop consonants are perceived categorically, whereas those made up of vowel distinctions are perceived continuously; that is, stimuli identified as belonging to the same category are discriminated almost as well as those perceived as belonging to different categories (see Repp, 1984, for a review).

One influential account of the differences in the perception of vowels and consonants is Fujisaki and Kawashima's (1970; see also Pisoni, 1973, 1975) dual-code theory. This theory assumes that two memory traces, an auditory and a phonetic trace, are constructed when a listener hears a speech sound. In order to discriminate between two speech sounds, the listener compares the phonetic traces of the two stimuli first and, if they are different, is able to make the discrimination. However, if the phonetic traces are the same, meaning that the two stimuli be- long to the same category, the listener must rely on the auditory trace to discriminate between the stimuli. The observed categorical perception of consonants versus the relatively continuous perception of vowels (Pisoni, 1975) is explained by assuming that the auditory store has difficulty holding the transient information identifying the consonant and thus can rely only on phonetic identity to make a decision. In contrast, longer lasting information for vowels, which is available in the auditory store, is more durable and can be used to make within-category discriminations.

The explanation for performance differences between vowels and consonants in the categorical perception paradigm is similar to the explanation for analogous differences in the memory paradigm. Vowels have an advantage over stop consonants because the auditory information is still present in some sort of sensory code, resulting in two possible codes that can be used to identify the item. This type of explanation has been given some additional support by research that shows nearly categorical responding, poor discrimination, and poor serial recall for very short synthetic vowels (e.g., Crowder, 1973a; Pisoni, 1973).

Subsequent theories have focused more on the relative discriminability of the items in the set to account for the above effects, although these views can also be interpreted as trying to specify more exactly what is different about vowels and consonants and why one might be represented more accurately or for longer than the other. For example, Ades (1977) found that there were many more justnoticeable-differences between pairs of vowels than between pairs of stop consonants. He concluded that differences in categorical perception could be explained merely by the range of values being compared instead of any hypothetical memory codes. In his view, the differences between discrimination and identification for vowel and stop-consonant continua can be explained in terms of general psychophysical principles that hold for nonspeech stimuli as well: The smaller the range of stimuli tested, the more the discrimination functions resemble the identification functions (the definition of categorical perception).

Similarly, Darwin and Baddeley (1974) used acoustic discriminability to explain the differences between serial recall of vowel- and consonant-contrasting lists. They suggested a degraded tape-recording model of auditory memory: Information in auditory memory degrades over time and the distinctions between consonants, because they are less discriminable than vowels, are destroyed at a faster rate, which leads to poorer memory for stop consonants. They tested this supposition by presenting subjects with lists of more discriminable consonants (/ash/,/am/,/ag/, for example) and similar vowels (/bI/,/be/,/bæ/) and found increased recency in the former and decreased recency in the latter conditions. They concluded that discriminability among the items in a set is highly correlated with how well they will be remembered.

All of the views mentioned so far relegate acoustic information to a more or less supplementary role, and, particularly for the memory theories, to a different structure entirely. An alternate view uses the idea of featural overwriting but incorporates the acoustic information in a much 
more central manner. Nairne $(1988,1990)$ developed a feature model of immediate memory that directly addresses a reason for the differential mnemonic affordances of vowels and stop consonants. The model was designed to account for the major effects observed in immediate memory settings, including the recency effect, the modality effect, the terminal and preterminal suffix effects, and effects of articulatory suppression, temporal grouping, and phonological similarity. It has recently been extended to account for word-length effects (Neath \& Nairne, 1995).

Nairne (1990) distinguished between two types of features that constitute a memory trace: Modality-dependent features represent the conditions of presentation, including presentation modality, whereas modality-independent features represent the nature of the item itself and are generated through internal processes such as categorization. Rather than placing the locus of echoic memory in a separate structure, such as the views discussed above, Nairne followed Watkins and Watkins (1980) in that echoic (i.e., physical) and nonechoic representations of an item are viewed as different aspects (or features) of a common memory trace. The feature model also accommodates other modalities. For example, the tactile modality produces both modality (Nairne \& McNabb, 1985) and suffix effects (Watkins \& Watkins, 1974), and the feature model explains these in the same way as auditory modality and suffix effects.

Nairne (1990) assumed that recall of an item will depend on the match between a degraded trace in primary memory and a particular undegraded trace in secondary memory. Thus, a major function of primary memory is to serve as a means of constructing and maintaining cues that may indicate which secondary memory trace was present on a particular list. Memory traces are represented by vectors of features; typically, simulations assume that there are 20 modality-independent features and either 2 (visual) or 20 (auditory) modality-dependent features (see Nairne, 1988,1990 , for further discussion). The actual values used for each feature are generated randomly for each trial from a small set of permissible values, typically $+1,-1$, and 0 .

Forgetting in the model is due primarily to the overwriting of particular features. Modality-dependent information can interfere only with modality-dependent features, whereas modality-independent information can interfere only with modality-independent features. If feature $i$ of item $n$ is the same as feature $i$ of item $n+1$, then feature $i$ of item $n$ is set to 0 with some probability, $F$. Typically, this probability is set at 1.0 , which means that featural overwriting always occurs. Thus, forgetting at this stage is implemented as a particular form of retroactive interference and is not dependent on time per se. It is important to note, however, that it is not the loss of trace information in and of itself that lowers recall; rather, it is the reduction in similarity of a given degraded trace to its undegraded trace relative to the similarity of the other items to the same trace.

Because it is the relative similarity of the degraded trace to the undegraded trace that primarily determines whether an item will be correctly recalled, the absolute number of modality-independent features does not affect performance very much (Neath \& Nairne, 1995). Rather, the range of values used to define each particular feature plays a far more important role because the larger the range, the less overwriting and the more discriminable each item (Neath, 1994). According to the feature model, there should be no differences in the number of modality-dependent features for vowels and stop consonants (Nairne, 1988). However, there should be a difference in how useful those features are. The features specifying stop consonants will be very similar to one another, resulting in increased similarity (as defined by the model) and consequently worse memory for those items than for vowels.

One common aspect of all of the theories discussed above is that they predict that greater discriminability will lead to better memory: As the amount of auditory information declines (Darwin \& Baddeley, 1974; Fujisaki \& Kawashima, 1970; Crowder \& Morton, 1969; Pisoni, 1973), or as the similarity of the modality-dependent traces increases (Nairne, 1990), the information necessary to discriminate between stop consonants is more likely to be lost than the information necessary to discriminate between vowels. Thus, the information needed to recall vowels is more durable or is less susceptible to interference than that specifying stop consonants. All of these views, then, relate the recall of items to the identification of the items; the easier the items are to identify, or the more discriminable the items, the better the level of recall.

One difficulty in evaluating this prediction is that direct comparisons between vowels and stop consonants are problematic. Vowels and consonants differ on many dimensions, including length, rate of change of the formants, and so on. What is needed is a comparison that includes stimuli that result in the same phonetic label as vowels (Strange, Jenkins, \& Johnson, 1983) but at the same time are similar to stop consonants in terms of the length and rate of spectral change. Surprenant and Speer (1991) showed that when syllables were tested that fell midway between vowels and stop consonants in terms of length and rate of change (e.g., liquids, nasals, and glides), memory performance also fell between that of vowels and consonants. These types of stimuli also tend to fall between vowels and stop consonants in a categorical perception task: In general, they are perceived less categorically than stop consonants but not as continuously as vowels (see Repp, 1984). However, these stimuli very likely also fall midway between vowels and stops in terms of their overall discriminability.

Because of these problems, we included a different kind of stimulus called a silent-center vowel 2 in the present experiments. These syllables are made by digitally editing consonant-vowel-consonant (CVC) syllables so that most of the center part of the vowel is replaced with silence. This results in syllables that consist of an initial and final stop consonant and the rapidly changing formant transitions into and out of the vowel, but they have virtually no steady-state information. Strange et al. (1983) developed these stimuli in order to support Strange's (1987) dynamic theory of vowel perception and found that these stimuli were identified almost as well as full CVC syllables. They have many vowel-like attributes, yet at the same time con- 
sist mainly of rapidly changing dynamic information of short duration. For example, Fox (1989), using a synthetic CVC series, showed that silent-center syllables are perceived categorically, like stop consonants. That is, although the steps between adjacent tokens are equal, there is a sharp discontinuity in category membership, and discrimination of the stimuli is very good across the category boundary and very poor for stimuli within the category. These stimuli therefore seemed to be like stop consonants on some dimensions yet like vowels on others.

\section{EXPERIMENT 1}

Experiment 1 was designed to examine the relationship between the discriminability of the vowels, consonants, and silent-center vowels, and serial recall of the same items. Three sets of six CVC stimuli were created. The "vowels" had the same initial and ending consonant, but varied the vowel; the "consonants" had the same vowel and ending consonant, but varied the initial stop consonant; and the "silent-center vowels" were identical to the vowels except that the steady-state portion of the acoustic signal was digitally removed. Subjects performed the identification task before performing the serial recall task.

\section{Method}

Subjects. Twenty-four Purdue University undergraduates volunteered to participate in exchange for credit in introductory psychology courses. All subjects were native speakers of American English.

Stimuli. The stimuli were produced by a female native speaker of American English in a sound chamber and were digitized at a $22.2-\mathrm{kHz}$ sampling rate. The vowel-contrasting tokens were $/ \mathrm{bab} /$, $/ \mathrm{bib} /, \mathrm{blb} /, / \mathrm{bEb} /, / \mathrm{bob} /$, and $/ \mathrm{bVb} /$. The consonant-contrasting tokens were $/ \mathrm{bæb} /$, /dæb/, /gæb/, /kæb/, /pæb/, and /tæb/. The silentcenter tokens were created from the vowel-contrasting tokens by removing all but four pitch pulses on either side of the vowel and replacing that information with silence (see Fox, 1989, for more details of this procedure). Each token was contained in an envelope lasting $500 \mathrm{msec}$.

Design. All stimulus events were under the control of an Apple Macintosh computer. The experiment was divided into three blocks, each block using one type of stimulus set (vowels, consonants, silentcenter vowels). Within each block, there were 24 identification trials followed by 15 serial recall trials. The identification trials tested each token four times, in random order. First, the computer played a sound, and then it displayed six buttons on the screen that contained the six possible responses. Using a mouse, subjects clicked on the button containing the name of the token they thought they had heard They had approximately $3 \mathrm{sec}$ in which to make a response, and the computer informed them of the correct response. The serial recall trials tested a random order of the six tokens on each trial. The tokens were presented at a rate of two per second and immediately following the offset of the sixth token, six buttons appeared on the screen. Using a mouse, subjects clicked on the buttons containing the names of the tokens in the order in which the tokens were played. Approximately $10 \mathrm{sec}$ were allowed for serial recall. The order of the blocks was completely counterbalanced over 6 subjects.

Procedure. Subjects were informed that we were interested in how accurately they could perceive and remember various sounds The two tasks were explained, and no subject reported any difficulty in using the mouse to respond. After subjects indicated that they understood the task, the first block began. Prior to the first trial of each block, a message reiterated the basic instructions for that particular task (e.g., identify the tokens or recall their presentation order). There were subject-paced pauses between each task, and between each block. All stimuli were presented over Sony MDR V5 headphones, and subjects were allowed to adjust the intensity to a comfortable level. Subjects were tested either individually or in pairs.

\section{Results and Discussion}

For all reported experiments, the analyses of main interest were comparing identification and overall recall performance for the vowels, consonants, and silent-center syllables. Comparisons used a Tukey HSD test, and differences were judged reliable as $p<.05$.

The overall proportions of vowels, consonants, and silentcenter syllables correctly recalled (collapsed across serial position) were $.48, .37$, and .28 , respectively. All of these values differed reliably from one another. There was no difference in identification of the vowels (.93) and consonants (.96), but both of these were reliably better than identification of the silent-center syllables (.51). The top panel of Figure 1 shows these results. Thus, in terms of identification, the vowels and the consonants were approximately equal, but there were probably ceiling effects, and although identification of the silent-center vowels was poor, it was still reliably above chance levels (.167).

As can be seen in the bottom panel of Figure 1, Experiment 1 replicated the basic findings of better serial recall of stimuli that differ in vowels than stimuli that differ in consonants over serial position (Cole, 1973; Crowder, 1971). A 3 (token type: vowels, consonants, or silent-center vowels) $\times 6$ (serial position) analysis of variance (ANOVA) on the serial recall data revealed reliable effects of stimulus type $\left[F(2,46)=32.76, M S_{\mathrm{e}}=0.043, p<.01\right]$, serial position $\left[F(5,115)=80.56, M S_{\mathrm{e}}=0.018, p<.01\right]$, and a reliable interaction $\left[F(10,230)=7.96, M S_{\mathrm{e}}=0.14, p<.01\right]$. Vowels were recalled better than consonants, and this difference was particularly large for the last one or two serial positions. Recall of the silent-center syllables was poor at all positions.

\section{EXPERIMENT 2}

Although both vowels and consonants were identified and recalled better than silent-center vowels in Experiment 1 , relatively little can be said about the relationship between discriminability and serial recall because of the high level of identification of the vowels and consonants. Beginning with Experiment 2, we added noise to the vowel and consonant tokens to reduce their discriminability, as measured by the identification task.

\section{Method}

Subjects. Twenty-four new subjects from the same pool as that in Experiment 1 volunteered to participate in exchange for credit in introductory psychology courses.

Stimuli. The stimuli were identical to those used in Experiment 1 except that noise was added to the vowel and consonant tokens. The same 500-msec sample of cafeteria babble from the CUNY nonsensesyllable test (Levitt \& Resnick, 1978) was digitally mixed with the original token to produce a new token. The base token was amplified by different amounts for the different stimuli in an effort to ma- 

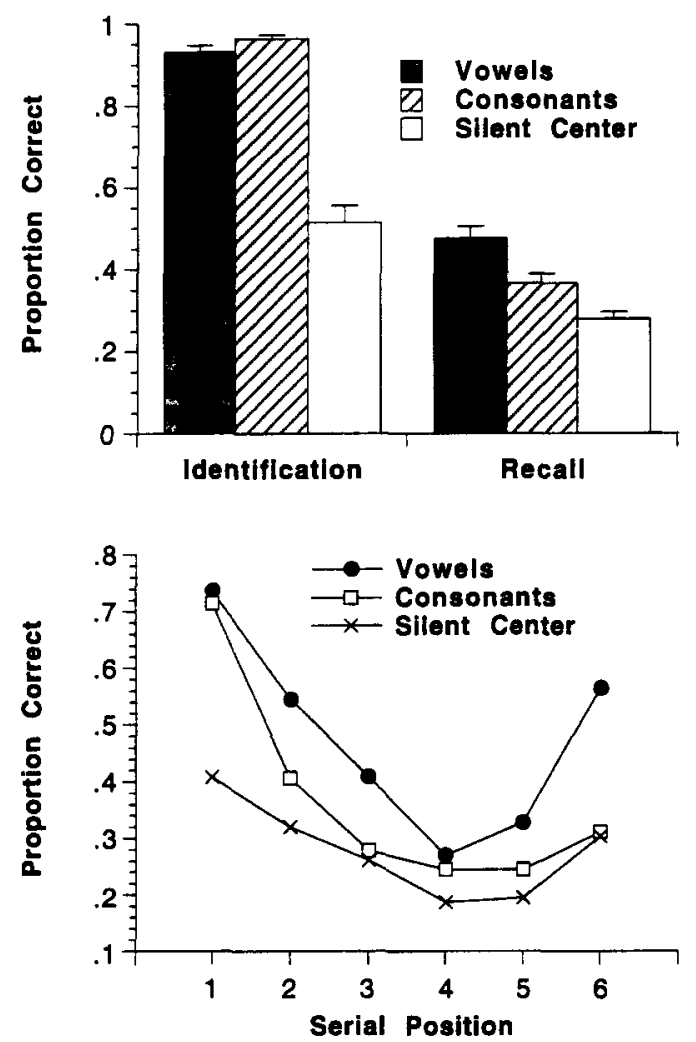

Figure 1. Proportion of items correctly identified and recalled by stimulus type (top), and proportion of vowels, consonants, and silent-center vowels correctly recalled as a function of serial position (bottom) in Experiment 1. Error bars show the standard error of the mean.

nipulate identification performance. The silent-center tokens did not have noise added and were thus unchanged from those in Experiment 1

Design. The design was identical to that in Experiment 1.

Procedure. The only procedural difference was that prior to each block, the six tokens were played once each to give the subjects experience with all six prior to the identification trials. This was accomplished by displaying the name of the token on the computer screen for $500 \mathrm{msec}$ while the appropriate token was being played.

\section{Results and Discussion}

As can be seen in Figure 2, adding the cafeteria noise reduced performance in both the identification and the serial recall tasks. There was no difference in identification between the consonants (.54) and silent-center syllables $(.52)$, and there was also no difference in overall recall of these items (.27 and .28, respectively). Identification (.76) and recall $(.41)$ of the vowels was reliably better than both of the other types of stimuli.

A 3 (token type: vowels, consonants, or silent-center vowels) $\times 6$ (serial position) ANOVA on the serial recall data revealed reliable effects of stimulus type $[F(2,46)=$ $\left.19.40, M S_{\mathrm{e}}=0.046, p<.01\right]$, serial position $[F(5,115)=$ $\left.37.34, M S_{\mathrm{e}}=0.016, p<.01\right]$, and a reliable interaction $\left[F(10,230)=5.59, M S_{\mathrm{e}}=0.14, p<.01\right]$. When performance on the identification task was equated for consonants and silent-center vowels, performance on the recall task was also equivalent. Identification of the vowels was superior to both, and recall was likewise superior. These results are entirely consistent with the common prediction made by all the theories of vowel and consonant differences mentioned earlier - that increased discriminability will lead to better serial recall performance.

\section{EXPERIMENT 3}

In Experiment 2, we were successful in equating identification performance of the consonants and silent-center syllables, and this resulted in equivalent recall performance. However, identification and recall of the vowels was still better than for the other two types of tokens. In Experiment 3 , we attempted to manipulate the discriminability of the vowels and consonants so that the consonants would be identified more easily than the vowels by manipulating the intensity of the noise added to the stimuli.

\section{Method}

Subjects. There were twenty-four new subjects from the same pool as that in Experiment 1.

Stimuli. The stimuli were identical to those used in Experiment 1 except that more noise was added to the vowel tokens than was added to the consonant tokens.

Materials, Design, and Procedure. The materials, design, and procedure were otherwise identical to those in Experiment 2.
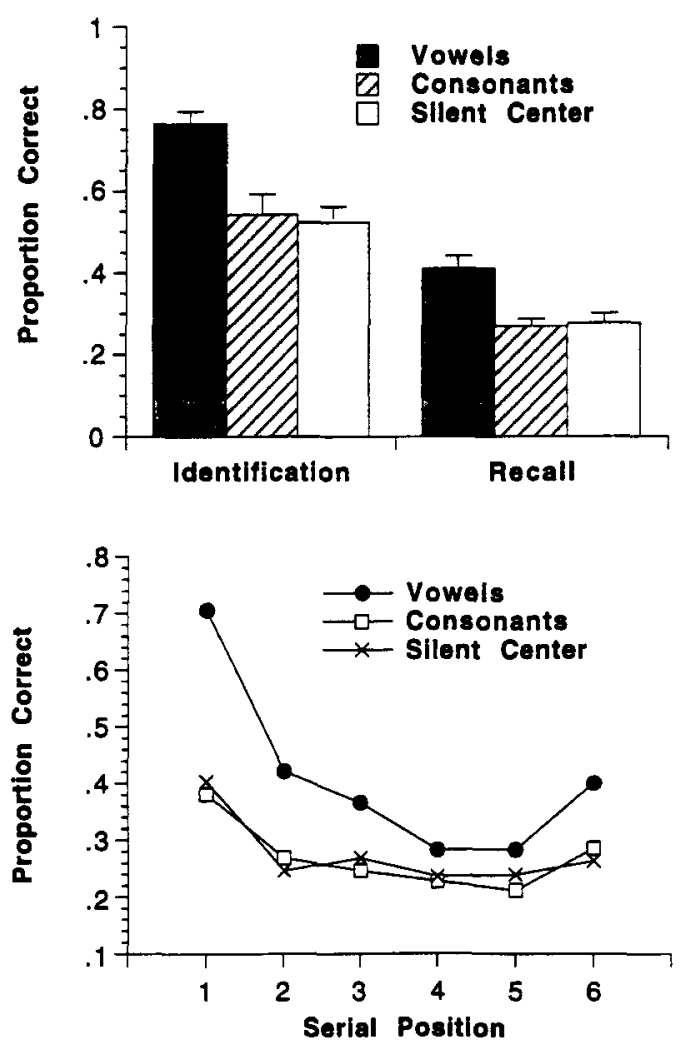

Figure 2. Proportion of items correctly identified and recalled by stimulus type (top), and proportion of vowels, consonants, and silent-center vowels correctly recalled as a function of serial position (bottom) in Experiment 2. Error bars show the standard error of the mean. 

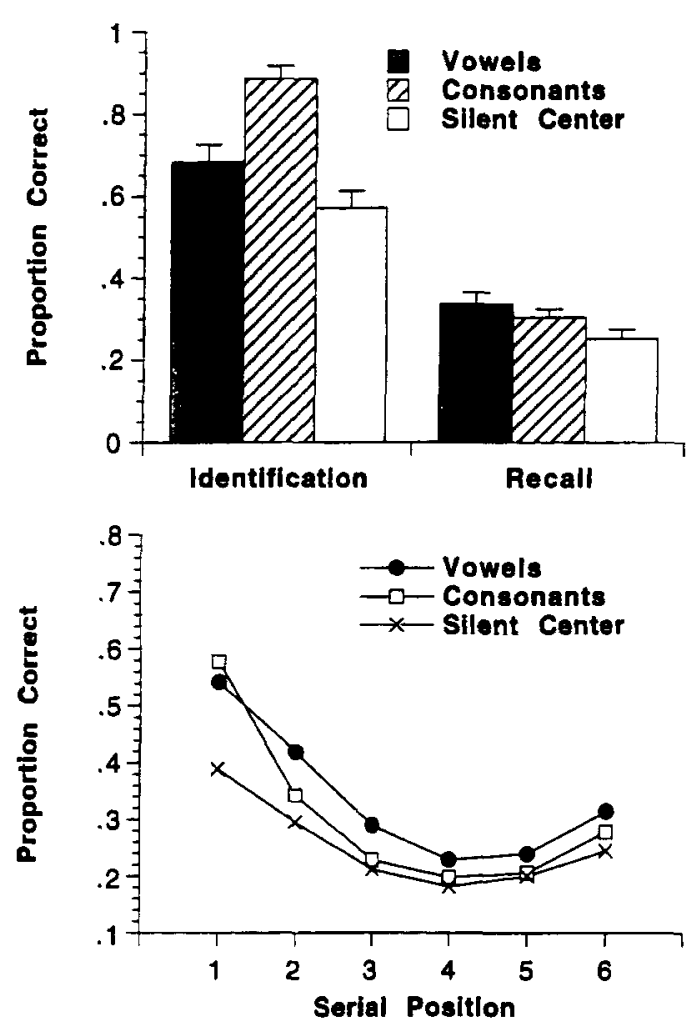

Figure 3. Proportion of items correctly identified and recalled by stimulus type (top), and proportion of vowels, consonants, and silent-center vowels correctly recalled as a function of serial position (bottom) in Experiment 3. Error bars show the standard error of the mean.

\section{Results and Discussion}

As can be seen in Figure 3, identification of the consonants $(.89)$ was reliably better than identification of the vowels (.68). However, recall of the vowels (.34) was slightly better than recall of the consonants (.30), although this difference was not reliable. Identification of the silent-center syllables $(.57)$ was reliably worse than identification of both full-syllable tokens, and recall of the silent-center syllables (.25) was reliably worse than recall of the vowels but did not differ reliably from recall of the consonants.

A 3 (token type: vowels, consonants, or silent-center vowels) $\times 6$ (serial position) ANOVA on the serial recall data revealed reliable effects of stimulus type $[F(2,46)=$ 4.36, $\left.M S_{\mathrm{e}}=0.061, p<.05\right]$, serial position $[F(5,115)=$ $\left.42.80, M S_{\mathrm{e}}=0.022, p<.01\right]$, and a reliable interaction $\left[F(10,230)=2.62, M S_{\mathrm{e}}=0.13, p<.01\right]$. Thus, the reliably better identification of the consonants compared with the vowels did not translate into better recall performance; although not reliable, recall of the consonants was slightly worse than recall of vowels.

\section{EXPERIMENT 4}

In Experiment 3, it was found that although identification of the consonants was better than that for the vowels, recall performance for consonants was equivalent or even slightly worse than that for the vowels. Experiment 4 was designed to produce higher identification performance on the silent-center vowels than on the full vowels. The method used for constructing the silent-center syllables was patterned after that of Fox (1989), who excised all except four pitch pulses on either side of the vowel. This was done in order to ensure that none of the steady-state information was available to help identify the vowel. However, in our first three experiments, the stimuli used were produced by a female talker, whereas Fox used formants appropriate for a male talker. Because females have a higher fundamental frequency (more pitch pulses or cycles per second), the four pulses occur over a shorter period of time, perhaps resulting in a greater proportion of the syllable being removed from the syllable in our stimuli. This might account for some of the difficulty our subjects were having identifying the silent-center syllables. Experiment 4 was designed to further test the recall advantage of vowels by using a new set of syllables, this time produced by a male native speaker of American English.

\section{Method \\ Subjects. There were 24 new subjects from the same pool as that in Experiment 1.}

Stimuli. The stimuli were identical to those used in Experiment 1 except that they were produced by a male native speaker of American English. Noise, from the same source as in Experiment 2, was again added to the vowel and consonant tokens.

Materials, Design, and Procedure. The materials, design, and procedure were otherwise identical to those in Experiment 2 with the exception that the number of identification trials was increased to eight per token, and the number of serial recall trials was increased to 20 per stimulus type.

\section{Results and Discussion}

As can be seen in Figure 4, identification of the silentcenter syllables $(.78)$ was reliably better than identification of the vowels (.68). However, recall of the vowels (.39) was slightly better than recall of the silent-center syllables (.36), although not reliably so. Both identification (.58) and recall (.28) of the consonants was reliably worse than that for the vowels and silent-center syllables.

A 3 (token type: vowels, consonants, or silent-center vowels) $\times 6$ (serial position) ANOVA on the serial recall data revealed reliable effects of stimulus type $[F(2,46)=$ $\left.11.20, M S_{\mathrm{e}}=0.047, p<.01\right]$, serial position $[F(5,115)=$ 64.81, $\left.M S_{\mathrm{e}}=0.014, p<.01\right]$, and a marginally reliable interaction $\left[F(10,230)=1.82, M S_{\mathrm{e}}=0.12, .05<p<.10\right]$. The silent-center vowels used here had a higher proportion of steady-state information remaining than those produced by the female talker. This led to better identification and recall performance, and it also minimized the differences between recall of the vowels and the silent-center vowels, hence the marginal interaction between syllable type and serial position. Even though they were identified better, however, recall of the silent-center vowels was not better than that for the vowels. 

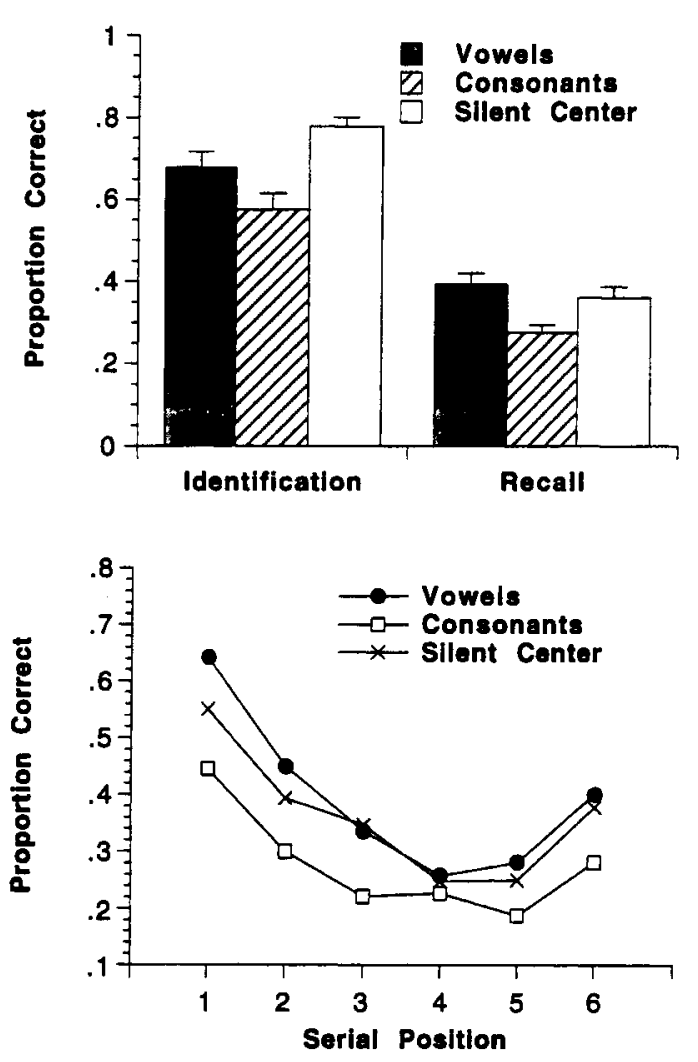

Figure 4. Proportion of items correctly identified and recalled by stimulus type (top), and proportion of vowels, consonants, and silent-center vowels correctly recalled as a function of serial position (bottom) in Experiment 4. Error bars show the standard error of the mean.

\section{SUMMARY OF EXPERIMENTS 1-4}

Experiment 1 reproduced the standard finding of better recall of vowels than consonants, but the identification performance could not be evaluated because of ceiling effects. When noise was added in Experiment 2, identification performance for consonants and silent-center syllables was equated and serial recall performance was also equivalent. In addition, identification of the vowels was better than identification of both the consonants and silentcenter syllables, and serial recall performance was also better. So far, this is consistent with the view that there is a strong relationship between the discriminability or identifiability of a set of stimuli and its level of recall.

However, in Experiment 3, identification of the consonants was better than identification of the vowels, but there was no reliable difference in recall performance. In fact, the difference was in the wrong direction: 34 for the vowels versus .30 for the consonants. Experiment 4 demonstrated a similar pattern, but this time with identification of the silent-center syllables being better than the identification of the vowels. As in Experiment 3, recall of the vowels was slightly, but not reliably, better than recall of the silent-center syllables (.39 vs. .36) even though they were identified less well. These findings are clearly prob- lematic for any view asserting that vowels are recalled better because of their increased discriminability.

\section{EXPERIMENTS 5-10}

Experiments 3 and 4 revealed findings problematic for any view that attributes better recall of vowels compared with consonants to increased discriminability. Experiments $5-10$ were designed to provide more data so that we could explore the relationship between identification and recall for each individual token at various levels of performance. Each experiment followed the previous experiments in terms of design, type and number of subjects, and procedure; the only difference was that we added noise at different intensities in a quasisystematic fashion in order to look at performance over a wider range of identification and recall levels. The goal of these five experiments, then, was to provide sufficient data to evaluate the prediction common to all theories of auditory memory: The relationship between discriminability and recall is linear, and increased recall is due to increased discriminability.

Experiments 5-8 used the same stimuli as those in Experiment 1 , but different amounts of noise were added to the stimuli in each of the new experiments. Experiments 9 and 10 used the same base stimuli as those in Experiment 4 , but again, different levels of noise were added.

\section{Method}

Subjects. There were 24 new subjects from the same pool as that in Experiment 1 in each of the six new experiments.

Stimuli. The stimuli were identical to those used in Experiment 1 (Experiments 5-8) or Experiment 4 (Experiments 9-10), except that noise was added to the vowel and consonant tokens and the base tokens were amplified by differential amounts to produce different levels of identification.

Materials, Design, and Procedure. The materials, design, and procedure were otherwise identical to those in Experiment 2 (Experiments 5-8) or Experiment 4 (Experiments 9-10).

\section{Results}

The data from Experiments 5-10 were combined with the data from Experiments 2-4. Figure 5 shows the proportion of times each individual token (e.g., /dæb/) was correctly recalled as a function of the proportion of times that same token was correctly identified. The silent-center data are not displayed because their identification was not systematically manipulated.

It is clear that there was a systematic relationship between discriminability (as measured by the identification task) and serial recall performance. The correlation for the vowels was .81, and for the consonants it was .80. Both the intercept and the slope of the best fitting linear regression differed for the two stimulus types, but the proportion of variance accounted for was basically identical. Although there was a systematic relation between identification of an item and its subsequent recall, it is clear that there were factors other than discriminability that gave rise to better recall of vowels than consonants.

Experiment 5 replicated the pattern of results from Experiment 2 in that identification and recall of the conso- 

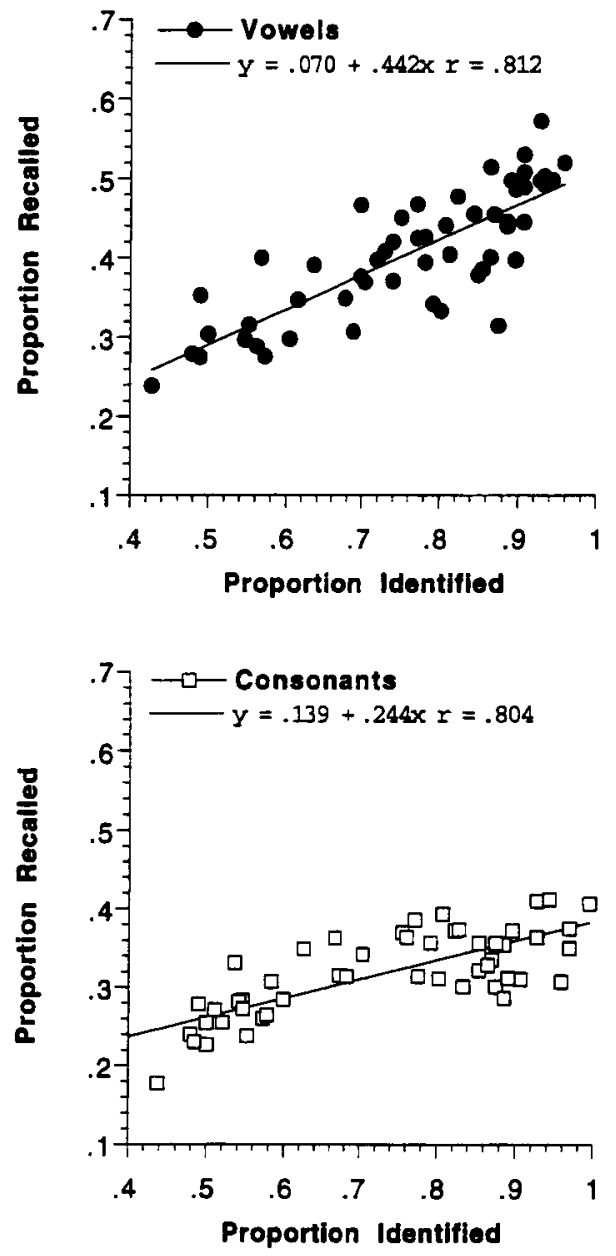

Figure 5. The proportion of individual vowels (top) and consonants (bottom) correctly recalled as a function of identification performance from Experiments 2-10.

nants (.55 and .28$)$ and silent-center syllables (.52 and .27) were equivalent. Experiment 6 replicated the pattern of results from Experiment 3 in that identification of the consonants $(.83)$ was reliably better than identification of the vowels (.69), but recall performance was equivalent (.36 for both types of stimuli). ${ }^{3}$

\section{EXPERIMENT 11}

Experiment 11 addressed one final issue. A great deal of previous research has shown that the judgment of one stimulus is highly dependent on the context in which the stimuli are presented (see Lockhead, 1992). For example, Repp and Crowder (1990) reported significant effects of the order of presentation of stimuli on vowel discrimination. The stimuli were judged differently depending on the immediate context in which they were presented. In all of our experiments, identification and recall trials were blocked by stimulus type. It is possible that performance on the identification trials could have been affected by changing the context in which the items were presented. Because we were interested in the connection between the discriminability of items and their memorability, we felt it would be important to determine what effect context might have on the identifiability of our sounds and how this might be related to the memory for those sounds.

In Experiment 11, therefore, the subjects received all of the stimuli randomly intermixed with all of the identification trials first, followed by the recall trials. Half of the subjects received the vowel and consonant stimuli embedded in noise and half received them with no noise. Hearing the syllables in an undistorted form may allow the memory for that item to be used as a prime or cue for identifying the same stimulus with the middle removed (silent-center syllables).

\section{Method}

Subjects. Forty-eight new subjects from the same pool as that in Experiment 1 were arbitrarily assigned to one of two groups.

Stimuli. The base stimuli were the same as those in Experiment 4.

Materials, Design, and Procedure. There were two differences between this experiment and Experiment 4 . First, on each identification trial, the token tested was randomly determined, and all of the identification trials occurred prior to the recall trials. In Experiment 4, the tokens were blocked by type. Second, half of the subjects received vowel and consonant tokens with no noise added, whereas the remaining half of the subjects received tokens with noise added. Even though the tokens were presented randomly rather than blocked by stimulus type, the identification and recall tests listed the names from only the appropriate stimulus type. For example, on any given identification trial, subjects could hear a vowel, a consonant, or a silentcenter vowel token. If they heard a vowel, the six buttons would be labeled with the six vowel possibilities; if they heard a consonant, then the six buttons would be labeled with the six consonant possibilities.

\section{Results and Discussion}

As can be seen in Figure 6, identification of the vowels and consonants was worse when noise was added to them than when there was no noise. More importantly, however, even though the silent-center syllables were physically identical in the two conditions, identification was reliably worse when the other tokens had noise (.58) than when the other tokens did not have noise (.72). Despite this effect of context on identification of silent-center syllables, there appeared to be no effect on the level of recall of the silentcenter syllables.

A 2 (context: noise added to the vowels and consonants versus no noise conditions) $\times 3$ (stimulus type) ANOVA on the identification data revealed a main effect of noise $\left[F(1,46)=83.79, M S_{\mathrm{e}}=0.053, p<.01\right]$, a main effect of stimulus type $\left[F(2,92)=7.11, M S_{\mathrm{e}}=0.014, p<.01\right]$, and an interaction $\left[F(12,92)=32.76, M S_{\mathrm{e}}=0.014, p<.01\right]$. This can be clearly seen in Figure 6: Identification of the silent-center syllables was better when there was no noise added to the vowels and consonants $[F(1,46)=5.14$, $\left.M S_{\mathrm{e}}=0.038, p<.05\right]$. This finding is interesting given that the silent-center tokens were physically identical in the two conditions.

For the recall data, there was also a main effect of noise $\left[F(1,46)=9.11, M S_{\mathrm{e}}=0.216, p<.01\right]$, of stimulus type $\left[F(2,92)=25.41, M S_{\mathrm{e}}=0.054, p<.01\right]$, and a main effect of serial position $\left[F(5,230)=134.48, M S_{\mathrm{e}}=0.018, p<\right.$ 
.01]. There was also a reliable interaction between noise and position $\left[F(5,230)=7.09, M S_{\mathrm{e}}=0.018, p<.01\right]$, and between stimulus type and position $[F(10,460)=2.70$, $\left.M S_{\mathrm{e}}=0.011, p<.01\right]$. The three-way interaction was also reliable $\left[F(10,460)=2.79, M S_{\mathrm{e}}=0.011, p<.01\right]$.

Of more interest was the interaction between noise and stimulus type $\left[F(2,92)=5.93, M S_{\mathrm{e}}=0.054, p<.01\right]$. Recall of the silent-center syllables did not change $[F(1,46)$ $<1]$ as a function of whether they were presented with vowels and consonants with noise (.39) or with vowels and consonants without noise (.40). There was an effect of serial position $\left[F(5,230)=43.71, M S_{\mathrm{e}}=0.014, p<.01\right]$, but there was no interaction between noise condition and position $[F(5,230)<1]$.

The results of Experiment 11, then, demonstrated that identification of a particular set of items, in this case silentcenter vowels, can vary depending on whether the context items are embedded in noise, but that this manipulation does not affect serial recall. Although identification of the silent-center syllables improved reliably when the vowel and consonant stimuli were presented with no noise, recall performance did not. It is important to note that during both the identification and recall test, the subjects knew the stimulus set (through the labels on response buttons), so differences cannot be attributed to between-set confusions. There was no doubt on the part of the subjects as to whether they had heard a vowel, a consonant, or a silent-center vowel, although there was some doubt as to the specific identity of the token.

\section{GENERAL DISCUSSION}

The majority of theories that address performance differences when the stimuli are vowels rather than consonants (e.g., Ades, 1977; Crowder \& Morton, 1969; Darwin \& Baddeley, 1974; Fujisaki \& Kawashima, 1970; Nairne, 1988,1990 ) predict that the more discriminable an item, the better the level of recall. In Experiments 2-10, noise was added to the vowel and consonant stimuli to avoid ceiling effects in identification performance. As Figure 4 clearly shows, the probability of correctly recalling an item was strongly influenced by that item's discriminability, as measured by the identification task. This finding supports the predictions of all the theories that recall of a stimulus is related to its discriminability.

However, the other important finding mandates a caveat that the relationship between recall and discriminability is far from perfect; indeed, it is possible to manipulate the items so that the opposite relationship results. For example, Experiments 3 and 6 showed significantly worse identification of vowel stimuli compared to identification of stop consonants, but recall of the vowels was slightly, though not reliably, better than that for the consonants. Similarly, Experiment 4 showed significantly worse identification of vowel stimuli compared to identification of silent-center vowels, but again, recall of the vowels was slightly better than recall of the silent-center vowels. Finally, Experiment 11 showed that manipulations, such as
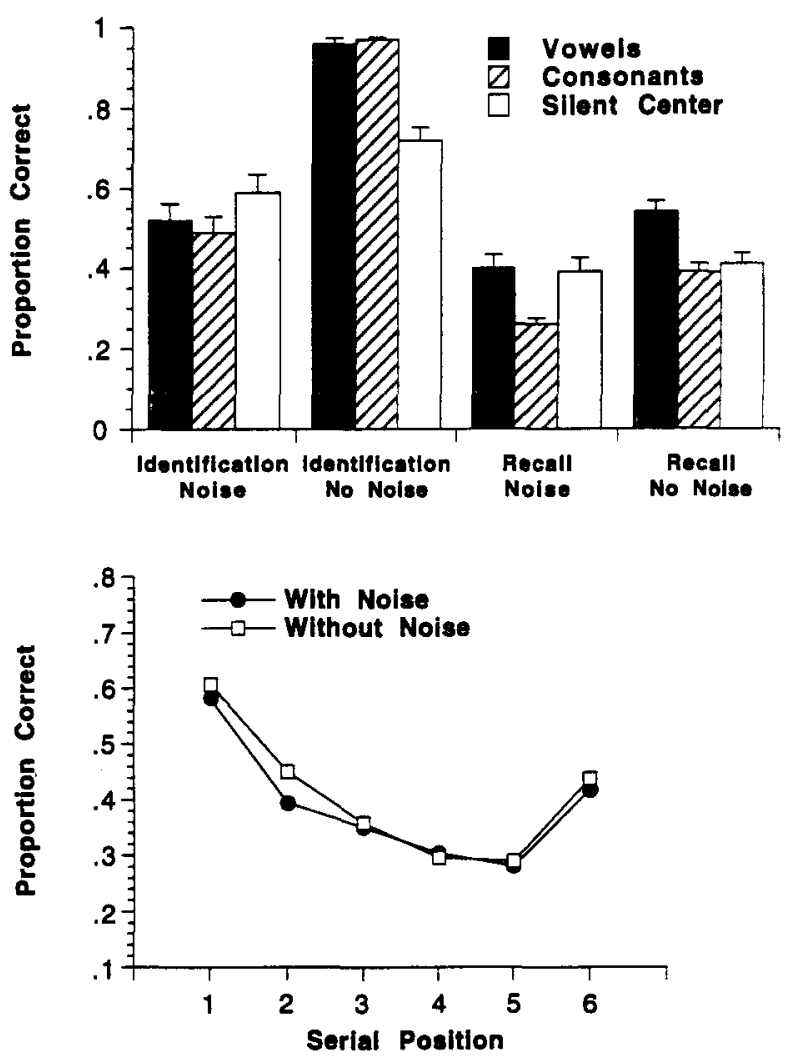

Figure 6. Proportion of items correctly identified and recalled by stimulus type as a function of noise condition (top) and proportion of silent-center vowels correctly recalled as a function of serial position and noise condition (bottom).

the presentation context, can affect the identification of a set of stimuli without affecting memory for those stimuli. This set of results seems paradoxical: In order to remember a stimulus, it is logically necessary to be able to identify it; however, worse identification of a stimulus can lead to better memory. How can this be explained?

One possibility might be that recall is determined by the relative discriminability of the memory representation rather than by the relative discriminability of the actual physical stimulus. Identification, on the other hand, rests almost entirely on the physical information available. This is one of the central aspects of Nairne's (1990) feature model. The modality-dependent features represent the conditions of presentation, including the acoustic information, whereas the modality-independent features are the result of the identification and categorization phases. According to the model, regardless of whether a token is presented visually or auditorily, the modality-independent features will be identical (Nairne, 1988). If the modality-dependent features are degraded, as when a token is embedded in noise, or if there are few modality-dependent features, as when a token is presented visually, successful recall will depend almost exclusively on the modality-independent features. In other words, it is the discriminability of the memory representation rather than the physical stimulus that is important. 
Note many verbal tokens will be positively correlated, as seen in Figure 5. But correlation does not imply causation. Consider Experiment 3, in which the consonants were identified better than the vowels, but were not recalled as well. According to the current view, the identification performance is based primarily on the modality-dependent features or the physical information presented to the subject. Because we added more noise to the vowels than to the consonants, the vowels should be less discriminable, and this was demonstrated in the identification data. However, for the recall task, a more durable code is required, such as the verbal label of the token (see, e.g., Conrad, 1964). For these particular stimuli, the names of the vowelcontrasting syllables are more discriminable than the names of the consonant-contrasting syllables. Even though subjects were not identifying the vowels as accurately as they identified the consonants, when they did, the codes that were created-which are reflected in the modality-independent features-were more discriminable. In this way, one can get better recall of more poorly identifiable stimuli.

Note also that this accords nicely with explanations of the basic modality effect. Visual presentation does not afford much useful modality-dependent information. Because of this, recall depends primarily on the modalityindependent information. With auditory presentation, there are more modality-dependent features, but they need not necessarily be useful. If the stimuli are easily discriminable physically, such as vowels, then this information will contribute to particularly robust recency effects. If, however, the stimuli are less discriminable, such as consonants or tokens in noise, then the modality-dependent information will be less useful. A prediction of the feature model, then, is that the less discriminable an item, the more the recall data should resemble recall data for visual presentation, with little or no recency effect evident. This is readily apparent in the bottom panel of Figure 2 .

Theories such as Crowder and Morton's (1969) PAS and Darwin and Baddeley's (1974) pure acoustic discriminability view cannot account for the apparent paradox that an item with low discriminability can be recalled as well as or even better than an item with higher discriminability. PAS requires that the information be precategorical, but the advantage according to the current analysis is due to the enhanced discriminability of the names rather than to the enhanced discriminability of the physical information. Darwin and Baddeley stated, "The usefulness of the information that can be retrieved from acoustic memory depends on the acoustic similarity of the items in the list" (p. 41).

In addition to explaining the apparent paradox, the current analysis also accords nicely with the literature on categorical perception. The identification task requires the subject to match a heard token with a stored standard. The discrimination task requires the subject to judge whether two heard tokens differ. According to the current view, categorical perception arises in the discrimination phase because the modality-dependent (physical) information is not as useful when the stimuli are consonants as when they are vowels. This is similar to the Fujisaki and Kawashima
(1970) view except that the feature model views all the information as part of a common trace.

The current view also predicts that stimuli can be constructed that will demonstrate categorical perception even though they are not speech stimuli. Several researchers (e.g., Cutting \& Rosner, 1974; Kewley-Port, Watson, \& Foyle, 1988; Miller, Wier, Pastore, Kelly, \& Dooling, 1976) have indeed reported this. We have already noted that shortened vowels are perceived more categorically than longer vowels (Crowder, 1973a; Pisoni, 1973). Finally, the current view predicts that adding noise to vowel stimuli should also produce more categorical perception (Lane, 1965).

\section{Summary and Conclusions}

Nairne's (1990) feature model can account for the major effects seen in immediate memory, including primacy, recency, and modality effects, terminal and preterminal suffix effects, the effects of articulatory suppression and phonemic similarity, and modality-specific temporal grouping effects. It can also account for the word-length effect, including interactions between word length, articulatory suppression, serial position, modality, and phonological similarity (Neath \& Nairne, 1995). The current analysis demonstrates how the feature model can also account for differences in recall and identification of vowels and consonants. Identification performance is determined primarily by acoustic discriminability, but recall is determined primarily by the discriminability of the memory representation, which in the current case is most likely the names of the stimuli. Although the discriminability of the memory representation will often be correlated with the discriminability of the acoustic information that specifies the stimulus, this relationship is not perfect and in fact can be reversed.

\section{REFERENCES}

Ades, A. E. (1977). Vowels, consonants, speech, and nonspeech. Psychological Review, 84, 524-530.

Ayres, T. J., Jonides, J., Reitman, J. S., Egan, J. C., \& Howard, D. A. (1979). Differing suffix effects for the same physical suffix. Journal of Experimental Psychology: Human Learning \& Memory, 5, 315-321.

COLE, R. A. (1973). Different memory functions for consonants and vowels. Cognitive Psychology, 4, 39-54.

Conrad, R. (1964). Acoustic confusions in immediate memory. British Journal of Psychology, 55, 75-84.

CROWDER, R. G. (1971). The sound of vowels and consonants in immediate memory. Journal of Verbal Learning \& Verbal Behavior, 10, 587-597.

Crowder, R. G. (1973a). Precategorical acoustic storage for vowels of short and long duration. Perception \& Psychophysics, 13, 502-506.

Crowder, R. G. (1973b). The representation of speech sounds in precategorical acoustic storage. Journal of Experimental Psychology, 98, 14-24.

Crowder, R. G. (1975). Inferential problems in echoic memory. In P. M. A. Rabbit \& S. Dornic (Eds.), Attention and performance $V$. New York: Academic Press.

CRowder, R. G. (1976). Principles of learning and memory. Hillsdale, NJ: Erlbaum.

Crowder, R. G., \& MORTON, J. (1969). Precategorical acoustic storage (PAS). Perception \& Psychophysics, 5, 365-373

Cutting, J. E., \& Rosner, B. S. (1974). Categories and boundaries in speech and music. Perception \& Psychophysics, 16, 564-570. 
Darwin, C. J., \& BADDELEY, A. D. (1974). Acoustic memory and the perception of speech. Cognitive Psychology, 6, 41-60.

DREWNOWSKI, A. (1980). Memory functions for vowels and consonants: A reinterpretation of acoustic similarity effects. Journal of Verbal Learning \& Verbal Behavior, 19, 176-193.

Fox, R. A. (1989). Dynamic information in the identification and discrimination of vowels. Phonetica, 46, 97-116.

FuJISAKI, H., \& KaWASHIMA, T. (1970). Some experiments on speech perception and a model for the perceptual mechanism (Annual Report of the Engineering Research Institute, Faculty of Engineering). Tokyo: University of Tokyo.

KeWLey-Port, D., Watson, C. S., \& Foyle, D. C. (1988). Auditory temporal acuity in relation to category boundaries: Speech and nonspeech stimuli. Journal of the Acoustical Society of America, 83, 1133-1145.

LANE, H. L. (1965). Motor theory of speech perception: A critical review. Psychological Review, 72, 275-309.

LECOMPTE, D. C. (1992). In search of a strong visual recency effect. Memory \& Cognition, 20, 563-572.

LEVITT, H., \& RESNICK, S. B. (1978). Speech reception by the hearing impaired: Methods of testing and the development of new tests. Scandinavian Audiology, 24, 29-54.

LoCKHEAD, G. R. (1992). Psychophysical scaling: Judgments of attributes or objects? Behavioral \& Brain Sciences, 15, 543-558.

Miller, J. D., Wier, C. C., Pastore, R. E., Kelly, W. M., \& Dooling R. M. (1976). Discrimination and labeling of noise-buzz sequences with varying noise lead times: An example of categorical perception. Journal of the Acoustical Society of America, 60, 410-417.

NAIRNE, J. S. (1988), A framework for interpreting recency effects in immediate serial recall. Memory \& Cognition, 16, 343-352.

NAIRNE, J. S. (1990). A feature model of immediate memory. Memory \& Cognition, 18, 251-269.

NaIRNE, J. S., \& Crowder, R. G. (1982). On the locus of the stimulus suffix effect. Memory \& Cognition, 10, 350-357.

NAIRNE, J. S., \& MCNABB, W. K. (1985). More modality effects in the absence of sound. Journal of Experimental Psychology: Learning. Memory, \& Cognition, 11, 596-604.

NEATH, I. (1994, May). Temporal encoding and the auditory superiority hypothesis. Paper presented at the 66th annual meeting of the Midwestern Psychological Association, Chicago.

NeAth, I., \& NaIRNE, J. S. (1995). Word-length effects in immediate memory: Overwriting trace decay theory. Psychonomic Bulletin \& Review, 2, 429-441

Neath, I., Surprenant, A. M., \& Crowder, R. G. (1993). The contextdependent stimulus-suffix effect. Journal of Experimental Psychology: Learning, Memory, \& Cognition, 19, 698-703.

PENNEY, C. G. (1989). Modality effects and the structure of short-term verbal memory. Memory \& Cognition, 17, 398-422.

PISONI, D. B. (1973). Auditory and phonetic memory codes in the discrimination of consonants and vowels. Perception \& Psychophysics, $13,253-260$

Pisoni, D. B. (1975). Auditory short-term memory and vowel perception. Memory \& Cognition, 3, 7-18.
REPP, B. H. (1984). Categorical perception: Issues, methods, findings. In N. J. Lass (Ed.), Speech and language (Vol. 10, pp. 243-335). New York: Academic Press.

REPP, B. H., \& Crowder, R. G. (1990). Stimulus order effects in vowel discrimination. Journal of the Acoustical Society of America, $\mathbf{8 8}$, 2080-2090.

SPOEHR, K. T., \& CORIN, W. J. (1978). The stimulus suffix effect as a memory coding phenomenon. Memory \& Cognition, 6, 583-589.

STRANGE, W. (1987). Information for vowels in formant transitions. Journal of Memory \& Language, 26, 550-557.

Strange, W. Jenkins, J. J., \& JoHnson, T. L. (1983). Dynamic specification of coarticulated vowels. Journal of the Acoustical Society of America, 74, 695-705.

SuRPRENANT, A. M. (1992). The mind's ear: Imagery for vowels and consonants. Unpublished doctoral dissertation, Yale University.

Surprenant, A. M., Pitt, M. A., \& Crowder, R. G. (1993). Auditory recency in immediate memory. Quarterly Journal of Experimental Psychology, 46A, 193-223.

Surprenant, A. M., \& SPEer, S. R. (1991, November). The influence of segmental sonority on immediate memory for syllables I. Poster presented at the 31 st annual meeting of the Psychonomic Society, Atlanta.

Watkins, M. J., \& Watkins, O. C. (1974). A tactile suffix effect. Memory \& Cognition, 2, 176-180.

Watkins, O. C., \& Watkins, M. J. (1980). The modality effect and echoic persistence. Journal of Experimental Psychology: General, 109, 251-278.

\section{NOTES}

1. Identification and discrimination are related terms, and the exact nature of the relationship depends on the specific task. For example, the categorical perception paradigm involves both within- and betweencategory comparisons. When a within-category comparison is made, a stimulus may be identified accurately even though it may not be discriminable from another stimulus, as, for example, when two stimuli that differ in VOT are given the same phonetic label. When a between-category comparison is made, correct identification implies correct discrimination, because the stimulus has been identified as having a different phonetic label than the other stimulus. In our experiments, only between-category judgments were required, and so correct identification can be seen as functionally equivalent to correct discrimination.

2 . In the speech perception literature these stimuli are usually called silent-center syllables rather than silent-center vowels. We use the latter term, however, to emphasize the type of information that is being varied and to be consistent with our other labels (e.g., "consonants" refers to lists with consonant-contrasting information, with the vowel held constant).

3. Experiments 7-10 did not provide any noteworthy results other than contributing a wide range of data points, as displayed in Figure 5.

(Manuscript received January 30, 1995; revision accepted for publication July 24,1995 .) 